\title{
Immunohistochemistry application as step one for diagnosing human intestinal spirochetosis
}

\author{
Sho Ogata ${ }^{1,2 *}$, Ken Shimizu ${ }^{2}$ and Kuniaki Nakanishi ${ }^{1,3}$ \\ ${ }^{1}$ Department of Pathology and Laboratory Medicine, National Defense Medical College, Tokorozawa, Saitama 359-8513, Japan \\ ${ }^{2}$ Department of Diagnostic Pathology, JCHO Saitama Medical Center, Saitama, Saitama 330-0074, Japan \\ ${ }^{3}$ Department of Laboratory Medicine, National Defense Medical College Hospital, Tokorozawa, Saitama 359-8513, Japan
}

\begin{abstract}
Diagnosing human intestinal spirochetosis (HIS), a colorectal infectious disease caused by Brachyspira species bacteria, in routine practice depends histologically on the presence of the so-called fringes on the mucosal epithelial surface. Cases in which hematoxylin-eosin (HE) sections raise suspicions of HIS may be further examined using immunohistochemistry (IHC). We aimed to elucidate whether an initial IHC application might increase the proportion of cases considered HIS-suspicious. Focusing on the fringes, we compared HIS diagnosed by HE (HIS-HE) and by IHC (HIS-IHC; based on results obtained using a polyclonal anti-Treponema pallidum antibody) in one month's consecutive lower GI endoscopic specimens in the pathology department at a single hospital. Among the 140 individuals examined during the period, $10 \mathrm{HIS}-\mathrm{IHC}$ cases (7.1\%; M:F=5:5; 34-75 yrs; median 57 yrs) were diagnosed, while HE pathology found eight HIS-HE cases (the fringes were thick and distinct; 5.7\%) and two possible cases. All 8 HIS-HE cases matched HIS-IHC cases. However, the two possible HIS-HE cases were denied by IHC, while two of the HIS-IHC cases were overlooked by HE pathology. Among the 10 HIS-IHC cases, 8 cases had conventional adenomas, 1 sessile serrated adenoma/polyp, 2 microvesicular hyperplastic polyps, 3 goblet cell rich hyperplastic polyps, and 1 ulcerative colitis. Cases with goblet cell rich hyperplastic polyps were at a higher rate in HIS than in non-HIS cases $(p<0.01)$. In conclusion, an initial IHC application might detect more HIS cases in routine practice, and importantly it might help to establish a correct diagnosis of HIS.
\end{abstract}

\section{Introduction}

Human intestinal spirochetosis (HIS) is caused by colonization by Brachyspira species bacteria within the human large intestine [1,2]. In routine practice, diagnostic pathologists finding characteristic, but subtle, so-called fringes -- band-like structures caused by a perpendicular arrangement of the bacteria -- on the colorectal surface mucosa on hematoxylin \& eosin (HE) sections usually raise a suspicion of HIS. Ancillary stainings such as Giemsa, Gram, and Warthin-Starry stains may be added to confirm the diagnosis [1]. Recently, immunohistochemistry (IHC) with commercially available antibodies to Treponema pallidum (TP), another type of Spirochete, has been reported to exhibit IHC cross-reaction with these fringes, and pathologic diagnosis of HIS has been performed on the basis of a finding of the fringe formation in routine histology, sometimes supported by this IHC confirmation [3,4]. In routine practice, however, IHC application to materials is performed to determine HIS only when it is supported by HE pathology. Therefore, to elucidate whether an initial IHC application might increase the proportion of HIS cases, we compared diagnostic utility between HE (HIS-HE; focusing on the so-called fringes) and IHC (HIS-IHC; using a polyclonal anti-TP antibody).

\section{Materials and methods}

In the present study, we examined all the specimens taken endoscopically from the large intestines and received at the Department of Diagnostic Pathology, JCHO Saitama Medical Center (Saitama, Japan) consecutively during a one-month period within October 2016 and November 2016. Total number of cases was 140. The male:female ratio was 86:54, and median ages were 64 years overall (between 20 and 94 years), 63 years in men (20-94 years) and 65 years in women (24-86 years). Clinical manifestations and clinical diagnosis of these 140 cases were obtained from their pathology request forms. Among them, 24 cases were positive for fecal occult blood, 3 for hematochezia, and 7 for bowel-habit abnormality. Following clinical diagnosis, 29 cases underwent endoscopy for follow-up after polypectomy, 12 for follow-up after colorectal cancer operation, 8 for inflammatory bowel diseases [including ulcerative colitis (UC)], and 2 underwent screening endoscopy for malignancy in other organs. Such data were not available in 48 cases.

The median number of samples taken from the intestines was two, and only one sample was taken in 62 cases. From the formalinfixed paraffin-embedded blocks of these samples, two sections were provided, one being for an HE-stained glass slide and the other for IHC. These sections were evaluated separately as to whether fringes could be found or not.

In the HE study, cases providing one or more specimens that histologically exhibited a distinct, hematoxylinophilic fringe-formation on the luminal surface of the colorectal surface epithelium were considered to have HIS (HIS-HE cases). Equivocal fringe features --

*Correspondence to: Sho Ogata, Department of Pathology and Laboratory Medicine, National Defense Medical College, Tokorozawa, Saitama 359-8513, Japan, Tel: +81-42-995-1505; Fax: +81-42-996-5192; E-mail: sogata@ndmc.ac.jp

Key words: Brachyspira; goblet cell rich hyperplastic polyp; human intestinal spirochetosis; immunohistochemistry; large intestine; spirochete; Treponema pallidum

Received: July 21, 2018; Accepted: July 27, 2018; Published: July 30, 2018 
viz. 1) not feathery or somewhat granular, 2) not thick, 3) eosinophilic, or 4) not distinctive due to crush or electrocautery artifacts -- were not regarded as indicating fringes.

For IHC, we performed the polymer-peroxidase method [Histofine ${ }^{\circ}$ Simple Stain MAX PO (MULTI); Nichirei Bioscience, Tokyo, Japan] on deparaffinized sections of HIS cases. After pretreatment with the heatinduced epitope retrieval technique [involving boiling at $100^{\circ} \mathrm{C}$ for 40 minutes in HEAT PROCESSOR SOLUTON pH 6 (Nichirei Bioscience)], the polyclonal antibody for TP (Abcam, UK) was incubated for half an hour. In the evaluation of the IHC, cases providing one or more specimens that histologically exhibited an immunoreactive fringeformation on the luminal surface of the colorectal surface epithelium were considered to have HIS (HIS-IHC cases). We considered thick and feathery immunopositive bands on the surface epithelium to be fringes, while thin, weak, or granular immunopositivity on the surface epithelium was neglected.

In the statistical analysis, Fisher's exact probability test was performed and a two-tailed possibility $p<0.05$ was considered significant. This study was approved by the local ethics committee of the JCHO Saitama Medical Center [Approval number: No. 15-11 (July 9, 2015)].

\section{Results}

In the HE pathology, eight HIS-HE cases (5.7 \%) and two equivocal HIS-HE cases (1.4\%) were found. The two equivocal cases exhibited non-distinctive and focal fringe-like feathery structures, meaning that the possibility of the presense of HIS could not be ruled out. In the IHC study, ten cases $(7.1 \%)$ were considered to have HIS (i.e., HIS-IHC), and there were no equivocal cases in IHC. Eight of the HIS-IHC cases matched the eight HIS-HE cases (Figure 1A,B). The frequencies of HISHE and HIS-IHC cases did not differ statistically from each other $(p=$ 0.81). The remaining two HIS-IHC cases did not match either of the equivocal HIS-HE cases, and they were overlooked in HE pathology.

Among the HIS-IHC cases, there were 5 men and 5 women, and their median age was 57 years (34-75 years). Clinical manifestations and clinical diagnoses of the IHC-HIS cases were as follows: 2 cases exhibited fecal occult blood, 1 bowel habit abnormality (watery diarrhea), 3 follow-up after polypectomy, 1 inflammatory bowel disease (UC), and 2 data not available. These clinical characteristics were not statistically different from those of non-HIS-IHC group.
Out of a total of 23 samples from the 10 HIS-IHC cases, 17 (73.9 $\%)$ were considered to exhibit immunopositive fringes, while in the HE study 15 samples (65.2\%) were considered to exhibit distinctive fringes. These frequencies did not differ statistically $(p=0.76)$. In six HIS-IHC cases, only one sample was taken, and these all exhibited the immunopositive fringe. With regard to location among the 23 HISIHC samples, the numbers of samples exhibiting immunopositive fringes were 0 of one sample obtained from the ileum, $9(81.8 \%)$ of 11 samples obtained from the right-side large intestine (viz. the large intestine proximal to the splenic flexure), and 8 (72.7\%) of 11 samples obtained from the left (viz. the large intestine distal to the splenic flexure). Among these 23 samples, pathologic diagnoses were 12 conventional (tubular, tubulovillous, and villous) adenomas, with one of these adenomas containing an adenocarcinoma component, 1 sessile serrated adenoma/polyp (SSAP), 2 microvesicular hyperplastic polyps (MVHPs), 3 goblet cell rich hyperplastic polyps (GCHPs; i.e., localized mucosal thickening showing epithelial hyperplasia without serration, a lesion that Japanese pathologists refer to as "hyperplastic nodules"; Figure 1C), and 5 inflammation. Of the 10 HIS-IHC cases, 8 cases had conventional adenomas, 1 SSAP, 2 MVHPs, 3 GCHPs, and 1 inflammation (UC). Three cases had combined lesions (one case with conventional adenomas, MVHPs, and GCHPs; one case with conventional adenoma and MVHP; and the remaining one with SSAP, conventional adenoma, and GCHP). Compared to the non-HIS-IHC group (130 cases), the frequencies in the HIS-IHC group (10 cases) of conventional adenomas ( $8: 2$ vs. $87: 43 ; p=0.501)$, SSAPs (1:9 vs. $5: 125$; $p=0.364)$, MVHPs (2:8 vs. $7: 123 ; p=0.126)$, and inflammation $(1: 9$ vs. $29: 101 ; p=0.689)$ were not statistically different. However, the frequency of cases having GCHPs were higher among HIS-IHC cases than in the non-HIS-IHC group (3:7 vs. 4:126; $p<0.01$ ).

The two equivocal HIS-HE cases were both women. When ileal samples were excluded, the number of samples obtained from each of 2 cases was two. Histology in one case revealed both conventional adenoma in both samples, and in the other case inflammation in both samples. HE sections of both cases showed focally hematoxylinophilic and partially feathery structures on the surface mucosa, but these structures were not distinct and did not cover the surface diffusely (Figure 2A,B). These fringe-like structures were not immunoreactive to anti-TP antibody.
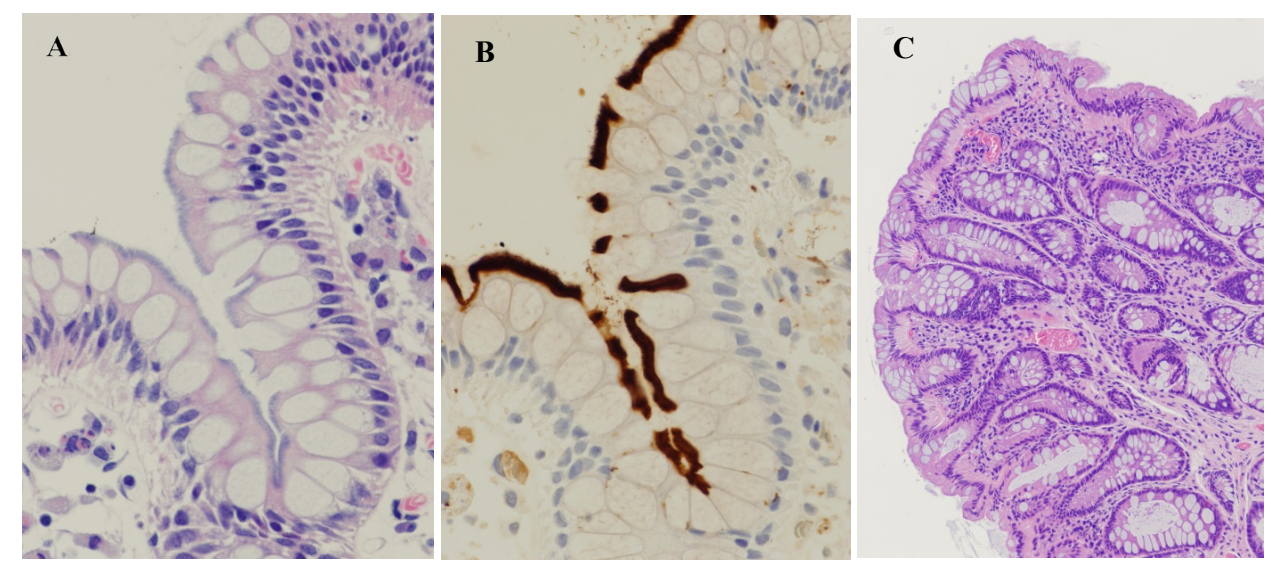

Figure 1. Representative images of the fringes and of a goblet cell rich hyperplastic polyp. A \& B. Histology revealed hematoxylinophilic, feathery structures (i.e., fringes) diffusely covering the luminal surface of the surface epithelium (A), while immunohistochemistry using anti-Treponema pallidum antibody revealed a thick, band-like, distinctive positive reaction at the same sites (B). C. Histology revealed a goblet cell rich hyperplastic polyp. Mucosal thickening with cryptal elongation and epithelial hyperplasia was observed, but crypts did not show serration. Such lesions are diagnosed as "hyperplastic nodules" in Japan. A \& C: hematoxylin \& eosin, A x400, C x100; B: diaminobenzidine, x400 
The number of overlooked HIS cases (i.e., HIS-IHC cases that were not also HIS-HE cases) was two, and the number of samples obtained from case each was one, with histology revealing conventional adenoma in both cases. One case showed a crush artifact in the fringe on the hyperplastic surface epithelium, but IHC exhibited a distinct immunoreactive band (Figure 3A,B). The HE section from the other case did not show distinct fringe structures, but the IHC section showed focal thick fringes accompanied by immunopositive spiral organisms within the attached mucus (Figure 3C-E).

\section{Discussion}

In the present study, we examined whether IHC with antiTP antibody could be usefully applied as the step-one method for diagnosing HIS. Although the boundaries of specimens tended to suffer from a nonspecific positive immunoreaction, IHC highlighted fringes on the mucosal surface by virtue of an intense immunoreaction even when no previous suspicion of HIS had been raised by HE pathology. All the HIS-HE cases were detected separately in the IHC study, as were two additional cases. A statistically significant difference in detecting power between $\mathrm{HE}$ and IHC was not found in the present study, and we suggest that IHC with anti-TP antibody could be used as a step-one method for detecting HIS in routine practice.

For diagnosing HIS, IHC pathology had some advantages over HE pathology, as follows: 1) cases that were overlooked by HE pathology were found by IHC, and 2) cases that were equivocal as regards HIS in

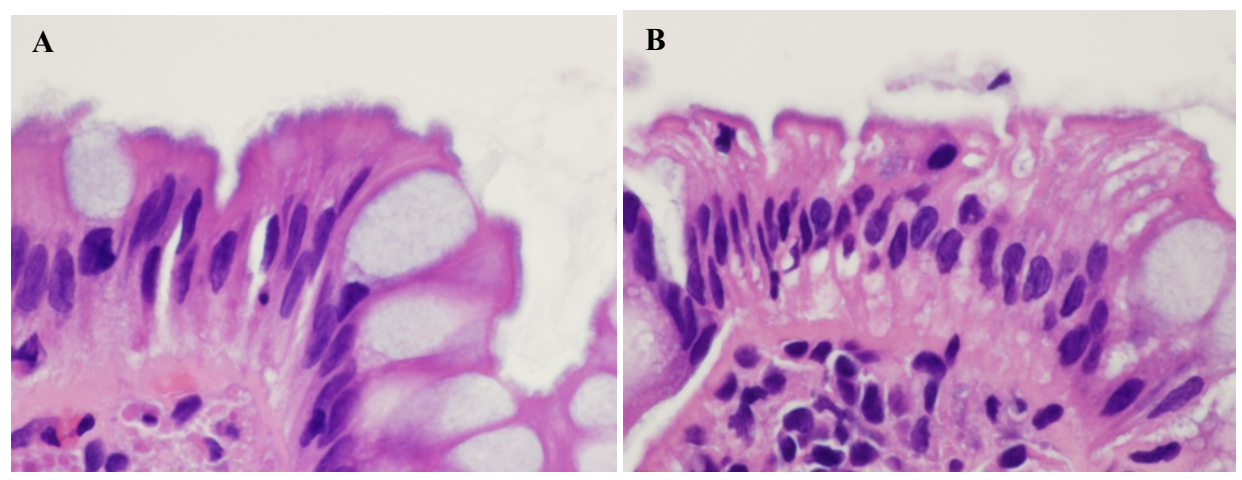

Figure 2. Equivocal cases in hematoxylin \& eosin pathology. A \& B. One case (A) included conventional adenoma, and the other (B) inflammation. Luminal surfaces of the colorectal surface epithelium in both specimens looked hematoxylinophilic and feathery, but not distinct and they were proved not to be fringes by immunohistochemistry (figures not shown). A \& B: hematoxylin \& eosin, x800
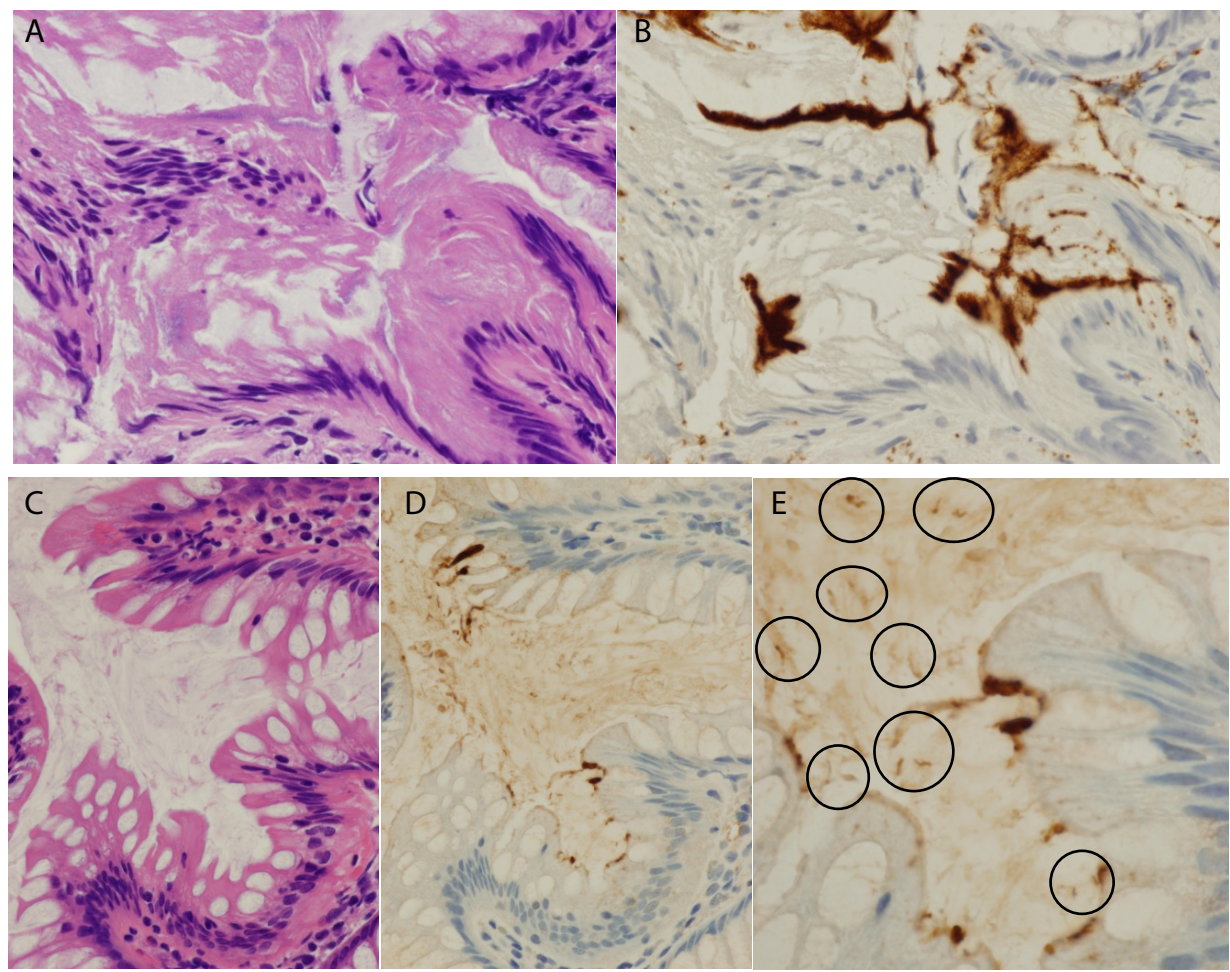

Figure 3. Overlooked cases by hematoxylin \& eosin pathology. A \& B. This case included conventional adenoma, and the non-neoplastic surface epithelium showed crush artifact, and hematoxylinophilic fringes looked slightly indistinct (A). However, immunohistochemistry (IHC) using anti-Treponema pallidum (TP) antibody highlighted distinct fringes (B). CE. The other case also included conventional adenoma. The non-neoplastic surface epithelium did not exhibit distinct fringes in the hematoxylin-eosin section (C), but IHC using anti-TP antibody revealed the focal presence of immunoreactive thick structures on the surface epithelium (D). Additionally, within the attached mucus, IHC also highlighted immunoreactive rods with a few spiral turns (circles in E), compatible with Brachyspira, the causative agent of human intestinal spirochetosis. A \& C: hematoxylin \& eosin, x400; B, D, \& E: diaminobenzidine, B \& D x400, E x 800 
the HE pathology were demonstrated by IHC not to have HIS. Actually, on the HE sections the fringes appeared as only subtle changes on the surface epithelium, and as such could be easily overlooked even by experienced pathologists. The overlooked cases ( 2 cases) in the present study were represented by only one sample per case, and the histologic sections revealed conventional adenoma in both cases. In our previous report, finding fringes within conventional adenoma components was difficult in HE pathology and only $24 \%$ of conventional adenoma exhibited fringes by IHC [5]. Possibly, background surface epithelium on which the fringe has formed may be included in conventional adenoma samples, although it may be a small volume and may suffer crush artifact or electrocautery damage. Indeed, crush was evident in one of the present overlooked cases. The explanation for the other overlooked case could be the fringes occupying a very small area being indistinct, resulting in it falling outside our strict critera for defining the fringe by HE pathology. This might raise a question as to whether the fringe that IHC detected in that case was real or not. However, the structure in the present overlooked case was considered to be a true fringe because of the coexistence of direct evidence of the existence of HIS in that case (immunopositive spiral organisms) within the attached mucus. In that regard, we previously reported finding immunopositive spiral organisms in the mucus of HIS cases at a very high rate in the imprint cytology of biopsy specimens and even in sections cut from formalin-fixed paraffin-embedded blocks $[4,6]$. Such overlooked cases may be inevitable when using HE pathology in routine practice.

However, using IHC, the key diagnostic structures are highlighted and easily found [4]. Furthermore, IHC is useful not only for morphologic detection of the fringes, but also for confirmation of the presence of bacterial antigens, at least to some degree. Although a superiority of IHC over HE pathology for diagnosing HIS was not established by the present study, applying IHC for this purpose may be recommended for the following reasons. First, it demonstrated that the two equivocal HIS-HE cases were not true HIS cases. Furthermore, the finding of spiral organisms within attached mucus by IHC lent strong support to a diagnosis of HIS. Possibly, in routine practice, although not for research, HE pathology may continue to be the first-line method for raising suspicion of HIS. In other words, IHC may not replace HE pathology, but instead become a good alternative to it, as step one for that purpose. However, for the purpose of investigating the clinical and pathologic features of true HIS, it is important to avoid equivocal cases, and strict criteria for the fringe (as applied in the present study) are essential for HE-alone pathology. For equivocal cases in HE pathlogy, further confirmation by IHC, nuclear acid analysis, or bacterial culture should be obtained.

The clinical manifestations and pathology of the HIS patients detected by HIS-IHC in the present study were as follows. The frequency of HIS in the IHC study was $7.1 \%$, a higher figure than in previous reports from Japan $[5,7,8]$. Although the present study has limitations -- 1) a single-center study, 2) only a one-month study, and 3) endoscopically taken specimens --, the higher rate may reflect a recent increasing trend of HIS in central Japan because a simultaneously obtained frequency in HE pathlogy was also relatively high. In the period of our study, we had the same number of women HIS cases as men cases. In our previous report on HIS, there were fewer women cases than men, using data from the same medical center [5]. Although this discrepancy might be due to the limitations mentioned above, careful investigation would be required to establish a whether recent change of mode in HIS has occurred between genders in Japan. Although HIS has been reported to be associated with certain lesions [5,9-11], the HIS cases in the present study did not show significant association with conventional adenoma, SSAP, or MVHP. However, HIS was associated with GCHPs that exhibited localized mucosal thickening and epithelial hyperplasia without serration. Mucosal thickening was reported in a previous study, and this may be one of the characteristics of HIS [12]. Although inflammation did not seem to be associated with HIS, a HIS case was found among the UC cases, representing $12.5 \%$ of UC cases in the present study. Previous studies have reported finding HIS among UC cases, and some HIS cases might mimic the clinical, endoscopic, and pathologic features of UC [13-15]. Considering the clinical importance of UC, the possible association between HIS and UC should be a subject for continued scrutiny.

\section{Conclusions}

IHC using anti-TP antibody was found to be effective for finding the so-called fringe formation on the surface epithelium, and on that basis should be useful as a step-one method for diagnosing HIS. Moreover, IHC should contribute to the correct diagnosis of HIS and to our understanding of its clinicopathologic characteristics.

\section{Acknowledgements}

The work was supported by JSPS KAKENHI grant number JP 18K07034. The authors also thank the staff of the Department of Diagnostic Pathology at JCHO Saitama Medical Center, Mr. Shinya Minabe, and Mr. Susumu Tominaga at National Defense Medical College for technical assistance.

\section{Conflict of interest}

None declared.

\section{References}

1. Rotterdam H (1997) Intestinal spirochetosis. In: Connor DH, Chandler FW, Schwartz DA et al editors. Pathology of Infectious Diseases, Stamford, CT: Appleton \& Lange 583-588.

2. Korner M, Gebbers JO (2003) Clinical significance of human intestinal spirochetosis-a morphological approach. Infection 31: 341-349. [Crossref]

3. De Brito T, Sandoval MP, Silva AG, Saad RC, Colaiacovo W (1996) Intestinal spirochetosis: first case reported in Brazil and the use of immunohistochemistry as an aid in histopathological diagnosis. Rev Inst Med Trop Sao Paulo 38: 45-52. [Crossref]

4. Ogata S, Shimizu K, Oda T, Tominaga S, Nakanishi K (2016) Immunohistochemical detection of human intestinal spirochetosis. Hum Pathol 58: 128-133. [Crossref]

5. Ogata S, Shimizu K, Nakanishi K (2015) Human intestinal spirochetosis: right-side preference in the large intestine. Ann Diagn Pathol 19: 414-417. [Crossref]

6. Ogata S, Higashiyama M, Adachi Y, Ohara I, Nishiyama J, et al. (2010) Imprint cytology detects floating Brachyspira in human intestinal spirochetosis. Hum Pathol 41: 249-254. [Crossref]

7. Tanahashi J, Daa T, Gamachi A, Kashima K, Kondoh Y, et al. (2008) Human intestinal spirochetosis in Japan; its incidence, clinicopathologic features, and genotypic identification. Mod Pathol 21: 76-84. [Crossref]

8. Sato H, Nakamura S, Habano W, Wakabayashi G, Adachi Y (2010) Human intestinal spirochaetosis in northern Japan. J Med Microbiol 59: 791-796. [Crossref]

9. Young JP, Price TJ, Moore J, Ruszkiewicz AR (2016) Human intestinal spirochetosis and its relationship to sessile serrated adenomas in an Australian population. Pathol Res Pract 212: 751-753. [Crossref]

10. Omori S, Mabe K, Hatanaka K, Ono M, Matsumoto M, et al. (2014) Human intestinal spirochetosis is significantly associated with sessile serrated adenomas/polyps. Pathol Res Pract 210: 440-3. [Crossref]

11. Calderaro A, Gorrini C, Montecchini S, Villanacci V, Bassotti G, et al. (2012) Intestinal spirochaetosis associated with hyperplastic and adenomatous colonic polyps. Pathol Res Pract 208: 177-180. [Crossref] 
Ogata S (2018) Immunohistochemistry application as step one for diagnosing human intestinal spirochetosis

12. Umeno J, Matsumoto T, Nakamura S, Yoshino S, Hirahashi M, et al. (2007) Intestinal spirochetosis due to Brachyspira pilosicoli: endoscopic and radiographic features. $J$ Gastroenterol 42: 253-256. [Crossref]

13. Iwamoto J, Ogata S, Honda A, Saito Y, Murakami M, et al. (2014) Human intestinal spirochaetosis in two ulcerative colitis patients. Intern Med 53: 2067-2071. [Crossref]
14. Chebli JM, de Abreu NC, Chebli LA, et al. (2016) Intestinal spirochetosis: an unusual cause of IBD flare-up during anti-TNF therapy. Int $J$ Colorectal Dis 31: 745-746. [Crossref]

15. Nishii S, Higashiyama M, Ogata S, Komoto S, Ito S, et al. (2018) Human intestinal spirochetosis mimicking ulcerative colitis. Clin J Gastroenterol 11: 145-9. [Crossref]

Copyright: $@ 2018$ Ogata S. This is an open-access article distributed under the terms of the Creative Commons Attribution License, which permits unrestricted use, distribution, and reproduction in any medium, provided the original author and source are credited. 